\title{
Experimental Evaluation of Channel Estimation and Equalisation in Non-Orthogonal FDM Systems
}

\author{
Waseem Ozan, Hedaia Ghannam, Tongyang Xu, Paul Anthony Haigh and Izzat Darwazeh \\ Department of Electronic and Electrical Engineering, \\ University College London, London, UK \\ \{w.ozan; hedaia.ghannam.15; tongyang.xu.11; p.haigh; i.darwazeh\}@ucl.ac.uk
}

\begin{abstract}
This paper describes the design and implementation of an experimental system created to evaluate the performance of channel estimation and equalisation for spectrally efficient frequency division multiplexing (SEFDM) systems in which higher spectral efficiency compared to conventional orthogonal frequency division multiplexing (OFDM) is achieved by violating the orthogonality of its subcarriers. This work proposes a new frequency-domain channel estimation and equalisation method, then investigates the employment of both OFDM and SEFDM pilot symbols for channel estimation to find channel state information (CSI). It is experimentally shown that the new method offers a reduction in the computational complexity compared to conventional time-domain estimation and equalisation for SEFDM systems with a similar system performance. The design of the baseband signal generation and signal detection using IFFT and FFT structure implemented using LabVIEW communication design suite is described in detail together with the baseband design of the system used to effect signal synchronisation and channel estimation and equalisation.
\end{abstract}

\section{INTRODUCTION}

Since the commercial deployment of $4 \mathrm{G}$ [1] networks, research into next generation networks has been developing rapidly with significant achievements [2]-[4]. This is due to the end user requirement for high data rates that support new media and data services. Thus, currently deployed $4 \mathrm{G}$ networks will offer insufficient rates to support such heavy data services. In future $5 \mathrm{G}$ networks, user demand is expected to be significantly in excess of the total network supply. As predicted in [5], over 50 billion devices will be connected to the internet by 2020 .

Although future 5G standard (or standards) are still not fixed, one of the most important requirements is higher spectrum efficiency. The two main propositions to achieve this are, first, reducing frequency spacing between adjacent carriers, such as in SEFDM [6], [7] systems, and second, send data faster, such as in FTN [8], [9] and TOFDM [10] systems. Generally, both of these methods will require non-orthogonal signals, which will either save bandwidth or time. However, by breaking the orthogonality rule, the systems performance is impacted in many ways. For example, one artefact of nonorthogonal systems is that inter-carrier interference (ICI) is generated in the signals that requires more complex receivers to detect the signals. Moreover, channel estimation and equalisation become more challenging due to the combined channel estate information (CSI) with ICI.

In this work, we investigate experimental implementation of channel estimation and equalisation of SEFDM signals in the frequency domain. In [11] and [12], channel estimation and equalisation were operated in the time-domain, since a standard single tap frequency domain estimator cannot work perfectly due to the self-induced ICI and this is also the case for equalisation. Although following this technique guarantees excellent channel estimation and equalisation, its complexity is rather high since matrix inversion and matrix multiplication operations are required. With such high complexity, implementation is not practical. Hence is the motivation of this work, to develop efficient channel estimation and equalisation method that work well in time variant fading channels yet have acceptable/practical implementation complexity. Therefore, this work proposes a novel frequency domain channel estimation and equalisation algorithm for the purpose of practical implementation.

This paper is organised as follows. Section II depicts SEFDM signal model. Section III explains the methodology of implementing frequency-domain channel estimation and equalisation. Section IV provides the description of the experimental setup in a realistic fading channel. The measured results and evaluate the system performance are shown in Section V. Finally, Section VI concludes the paper.

\section{SEFDM SIGNAL MODEL}

This section first outlines the mathematical signal model for SEFDM signals, then describes the methodology of SEFDM system implementation.

\section{A. Signal Model}

An SEFDM symbol consists of $N$ complex quadrature amplitude modulation (QAM) symbols denoted as $S$. Every QAM symbol is modulated on a non-orthogonal subcarrier. The overall continuous time SEFDM signal $x(t)$, consisting of $l$ SEFDM symbols is expressed as follows [12]:

$$
x(t)=\frac{1}{\sqrt{T}} \sum_{l=-\infty}^{\infty} \sum_{n=0}^{N-1} S_{l, n} \exp \left(\frac{j 2 \pi n \alpha(t-l T)}{T}\right)
$$

where $T$ is the SEFDM symbol duration, $\alpha=\Delta f T$ is the bandwidth compression factor, i.e. $\Delta f$ is the frequency spacing between two adjacent subcarriers, $N$ is the number of subcarriers in each SEFDM symbol, $S_{l, n}$ is the complex QAM symbol that is modulated on the $n^{\text {th }}$ subcarrier of the $l^{\text {th }}$ SEFDM symbol and $\frac{1}{\sqrt{T}}$ is the normalisation factor. Since the available data rate on each subcarrier is kept the same in comparison to the data rate of OFDM subcarriers, the distance between subcarriers in the frequency-domain is scaled down by $\alpha$, then the bandwidth saving is equal to $(1-\alpha)$ of the available bandwidth. When $\alpha=1$, an OFDM symbol is obtained, while for $\alpha<1$, the result is an SEFDM 


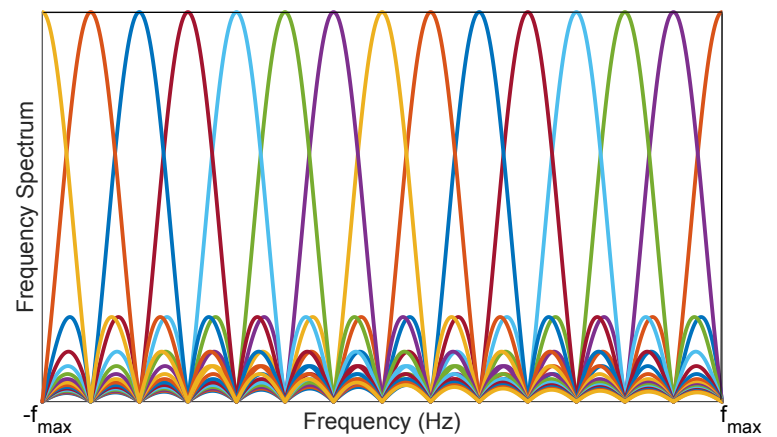

Fig. 1. OFDM frequency spectrum.

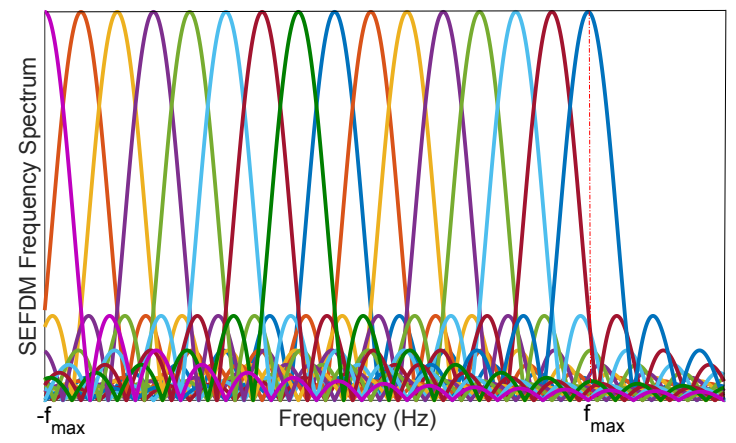

Fig. 2. SEFDM frequency spectrum.

symbol. Fig. 1 and Fig. 2 depict the OFDM and SEFDM signals.

Moreover, the discrete SEFDM signal can be simply given in matrix form as follows [12]:

$$
X=\boldsymbol{\Phi} S
$$

where $X=\left[x_{0}, x_{1}, \ldots, x_{Q-1}\right]^{T}$ represents a $Q$-dimensional vector of a sampled SEFDM symbol in the time-domain, $S=\left[s_{0}, s_{1}, \ldots, s_{N-1}\right]^{T}$ as an $N$-dimensional vector of a sampled input in the frequency-domain and $\Phi$ is a $Q \times N$ twodimensional matrix that signifies the sampled carrier matrix, where $Q=\rho * N$ and $\rho$ is the oversampling ratio. The $\boldsymbol{\Phi}$ matrix elements are $\boldsymbol{\Phi}_{k, n}=\frac{1}{\sqrt{Q}} \exp (j 2 \pi n k / Q)$. The symbol $[.]^{T}$ indicates the vector transpose.

Assuming a realistic scenario, i.e. the transmitted signal is passed through a wireless fading channel, $\hat{H}$; at the receiver, the channel-distorted signal is contaminated with additive white Gaussian noise (AWGN), $Z$ resulting in the received signal, which is then correlated with the complex conjugate carrier matrix, $\boldsymbol{\Phi}^{*}$. The reception process is expressed as [12]:

$$
R=\boldsymbol{\Phi}^{*} H X+\boldsymbol{\Phi}^{*} Z=\boldsymbol{\Phi}^{*} H \boldsymbol{\Phi} S+\boldsymbol{\Phi}^{*} Z
$$

where $R$ is the demodulated signal consisting of stream of symbols of $N$-dimensional vector size, and $Z_{\Phi^{*}}$ is the conjugate carriers correlated with the AWGN samples.

\section{B. Signal generation}

In this work, a single IFFT is implemented to realise SEFDM signal generation; where a specified number of zeros were added at the input of the IFFT, at the edges of the input vector, meaning that the number of samples at the input vector to the IFFT becomes $V=Q / \alpha$. Consequently, a $V$-point IFFT is required to generate the SEFDM signal of $N$ input subcarriers, where the remaining $V-N$ inputs are fed with zeros. Of which, $\gamma=Q-N$ zeros are introduced due to the oversampling ratio, $\rho$, while the $\Lambda=V-Q$ number of zeros to the input of IFFT and discarded samples of the output of the IFFT, are variable according to the bandwidth compression in SEFDM systems for SEFDM data and pilot symbols. In the case of using different compression values for SEFDM data symbols, $\alpha$, and SEFDM pilot symbols, $\alpha_{p}$, the discarded samples of the pilot symbols at the output of IFFT is $\Omega=Q / \alpha_{p}-Q$ while $\xi=\Lambda+\gamma-\Omega$ is the difference between the number of transmitted samples in SEFDM data and pilot symbols, as shown in the Fig.3.

\section{Channel Estimation and Equalisation}

The transmitted signals transversing a radio channel are impacted upon by the frequency response of the channel. After reception of the signals and, after undergoing the usual processes, transformation back into the frequency domain, channel estimation and equalisation processes are necessary before symbol de-mapping, in an attempt to eliminate the channel effects from the signal.

\section{A. Channel Estimation}

1) Channel estimation using OFDM pilots: In this method an OFDM pilot is applied. The OFDM pilot has subcarrier spacing equal to that of SEFDM but with a lower data rate at each subcarrier, resulting in the restoration of the orthogonality between subcarriers and absence of ICI and hence, understanding of the ICI. The time duration of an OFDM pilot symbol is longer than the time duration of the SEFDM symbols, resulting in slightly impaired spectral efficiency. Due to the orthogonal nature of OFDM signals, a simple estimation process can be used, that follows [13]:

$$
\hat{H}=S_{H} / S_{p}
$$

where $\hat{H}$ is the estimated channel characteristics at each active subcarrier using an OFDM pilot symbol, $S_{H}$ is the demodulated OFDM pilot that has been through the aforementioned fading/AWGN channel and $S_{p}$ is the expected received OFDM pilot symbol after the demodulation process, which is clearly the same transmitted pilot symbol, due to the fact that OFDM systems have no self-induced ICI between the subcarriers, as mentioned.

2) Channel estimation using SEFDM pilots: In this work, we propose a new channel estimation method using an SEFDM pilot to estimate the channel characteristics at the relative frequency positions of each subcarrier. The frequency spacing of these pilots are compressed, relative to those of an equivalent OFDM symbol of the same time duration. In SEFDM systems, the auto-ICI generated between subcarriers due to the bandwidth compression is deterministic and can be described mathematically by the correlation matrix as given in [14]. The receiver has the transmitted pilot symbols information and thus it applies the knowledge of the correlation matrix to find the expected received pilot symbols, $\hat{S_{p}}$ that are defined as $\hat{S}_{p}=C S_{p}$, where $S_{p}$ is the known transmitted pilot symbol and $C$ is the correlation matrix [14]. The $\hat{S}_{p}$ symbols are not equivalent to the transmitted symbols 

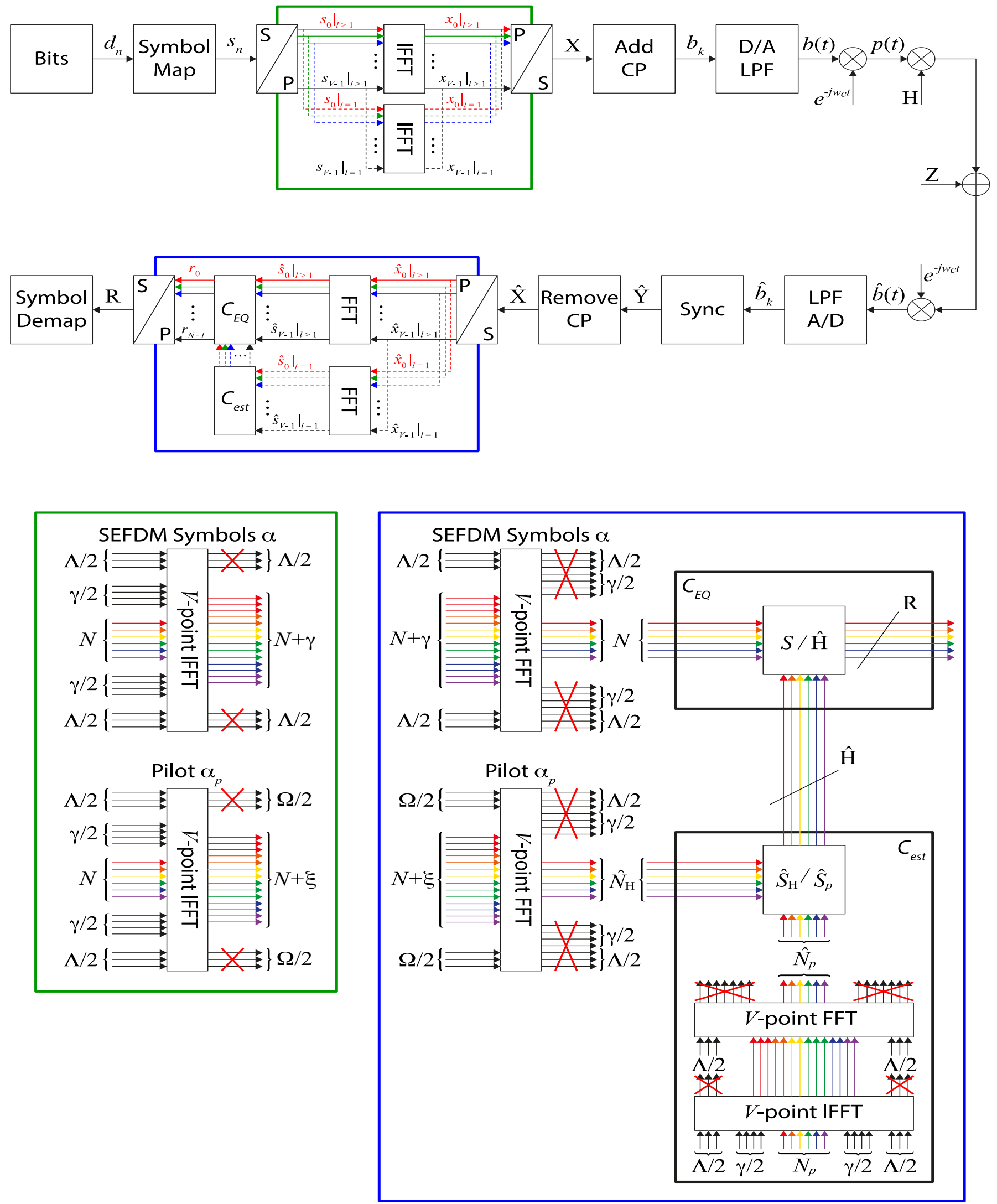

Fig. 3. schematic block diagram of SEFDM system

because of the interference effects even when no channel is present. The estimated channel characteristics are expressed as:

$$
\hat{H}=\hat{S_{H}} / \hat{S_{p}}
$$

where $\hat{H}$ is the estimated channel characteristics at each active subcarrier using the SEFDM pilot symbol, $\hat{S_{H}}$ is the demodulated SEFDM pilot that has been through a fading AWGN channel and $\hat{S}_{p}$ is the expected received SEFDM pilot 
symbol after the demodulation process.

\section{B. Channel Equalisation}

Channel equalisation is the task of reversing the channel response that affects the radio signals by using the CSI found by the channel estimator. The equalisation process is done by multiplying the received signal with the inverse of the channel characteristics and can be expressed as

$$
R=S / \hat{H}
$$

where $R$ is the equalised SEFDM data symbols and $S$ is the received SEFDM data symbols. The received symbols are affected by self interference that comes from the lack of non-orthogonality, delay spread that produce interference in the signal and AWGN from the electronic devices that are used to receive the signal. Channel equalisation utilise the estimated channel state information to reverse the channel effects. However, the self ICI generated in the SEFDM signal between the subcarriers can not be cancelled by the equalisation process, which means $R$ contains the self ICI. In order to tackle the problem of the ICI in SEFDM signals, several detection methods are proposed and implemented to recover the data after the equalisation process [15]-[20]. However, there is no detector implemented in this experiment because the focus of this work is to evaluate the channel estimation and equalisation processes.

\section{EXPERIMENTAL SETUP}

This section evaluates the channel estimation and equalisation techniques for different LTE channel emulation scenarios. In general, the testbed of this experiment consists of universal software radio peripheral (USRP) transceivers (NI USRP RIO N2395R) programmed using LabVIEW, plus, a Spirent VR5 channel emulator to generate realistic LTE channels. Both software and hardware specifications are detailed below.

\section{A. Experimental Setup \& Parameters}

The schematic block diagram is outlined in Fig. 3. At the transmitter, a stream of pseudorandom bits $d_{n}$ is generated, which are mapped into either binary phase shift keying (BPSK), quadrature phase shift keying (QPSK) or 16-quadrature amplitude modulation (16-QAM) format. Consequently, the data symbols $S_{n}$ are divided to $N$ parallel streams by a serial to parallel $(S / P)$ converter, where $N$ is the number of subcarriers in each SEFDM symbol. are fed to the SEFDM signal. The parallel streams are input to an inverse fast Fourier transform (IFFT) of length $V$.

It is important to emphasize that for the case of OFDM pilot, in order to maintain the orthogonality between its subcarriers while having the same frequency seperation of SEFDM, the data rate of each subcarrier has to be reduced by a factor of $(\alpha)$.

Then, the SEFDM samples are converted back to a serial stream $X$ by a parallel to serial $(P / S)$ converter. In order to reduce the effect of inter symbol interference (ISI) between adjacent symbols in a multipath wireless channel, a cyclic prefix (CP) is added at the beginning of each SEFDM symbol. In this experiment, the symbols are transmitted in frames, each of length fourteen SEFDM symbols with the first
OFDM/SEFDM symbol being reserved as a pilot. The frame bandwidth is $18 \mathrm{MHz}$ for OFDM while in SEFDM systems the occupied bandwidth is $(1-\alpha) * 18 \mathrm{MHz}$ using the same number of subcarriers. Each frame is of length fourteen SEFDM symbols with the first OFDM/SEFDM symbol being reserved as a pilot.

In the final stage at the transmitter, the digital complex signal $b_{k}$ is fed to an FPGA inside the national instrument (NI) USRP RIO N2953R of $120 \mathrm{MHz}$ channel bandwidth to perform digital to analog (D/A) conversion and frequency up-conversion to the $2 \mathrm{GHz}$ band. The experimental system specifications are shown in Table I

TABLE I

EXPERIMENTAL SYSTEM SPECIFICATIONS

\begin{tabular}{ll}
\hline Parameters & Values \\
\hline \hline Central carrier frequency & $2 \mathrm{GHz}$ \\
\hline Sampling frequency & $30.72 \mathrm{MHz}$ \\
\hline Signal bandwidth & $18 \mathrm{MHz}$ \\
\hline Values of $\alpha$ & $1(\mathrm{OFDM}) ; 0.9 ; 0.8 ; 0.7$ \\
\hline Subcarrier baseband bandwidth & $15 \mathrm{KHz}$ \\
\hline Subcarrier spacing & $\alpha \times 15 \mathrm{KHz}$ \\
\hline IFFT/FFT size & 2048 \\
\hline Cyclic prefix & 512 \\
\hline Modulation scheme & BPSK; QPSK; 16-QAM \\
\hline
\end{tabular}

\section{B. Realistic Fading Channel Model}

The RF signal is transmitted through a VR5 channel emulator that has LTE wireless channel model parameters [21], the output of VR5 is fed to the receiver USRP, which down-converts the analogue RF frequency to baseband signal and then converts the signal back to digital domain. A Schmidl and Cox [22] synchronization is applied in this experiment, where two identical timing sequences are added at the beginning of each frame to identify the beginning of the received frame $\hat{b_{k}}$. After down conversion, two sequences are correlated to get a correlation peak, which indicates the estimate of the starting sample of the data frame, $\hat{Y}$ for the analog to digital (A/D) converter.

Following this, CP is removed from the received symbols, then $\hat{X}$ is converted to parallel streams and fed to the lower FFT in the recovery block and then to the channel estimator block. The signal is received from different paths with different delays, thus it has a multi-delay spread equals to the difference between the longest path and line of sight. Channel estimation and equalisation are implemented to remove the phase and amplitude distortion. In order to estimate the channel effect using OFDM pilot, a standard one tap channel estimator is utilized. On the other hand, estimating the channel with SEFDM pilot requires a knowledge of self ICI generated in SEFDM systems. The $C_{\text {est }}$ block diagram in the lower left corner of Fig. 3 represents the process. The CSI defined as $\hat{H}$ is given to the equaliser to cancel the channel effect of the rest thirteen symbols in the same received frame, this process is depicted in the block $C_{e q}$.

\section{EXPERIMENTAL RESULTS}

\section{A. Channel Estimation Performance}

In this experiment, minimum square error (MSE) performance is practically examined to evaluate the frequencydomain channel estimation performance. In a previous work 


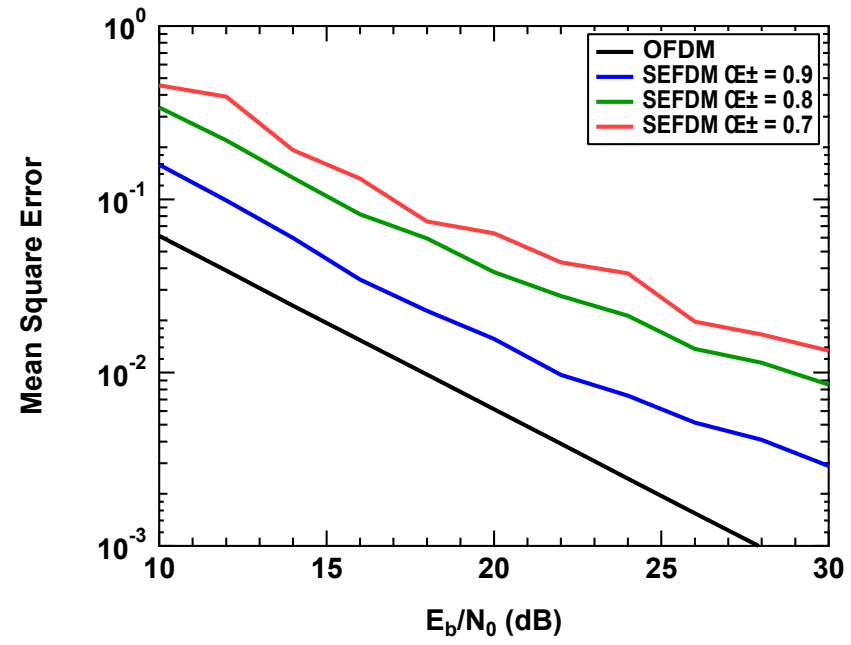

Fig. 4. Minimum square error versus $\mathrm{Eb} / \mathrm{No}$

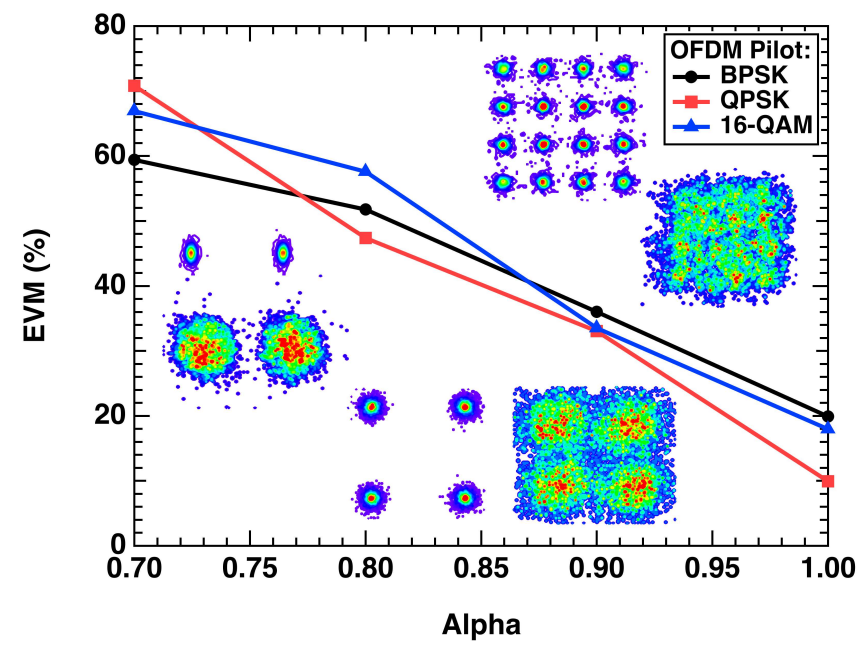

Fig. 5. Error vector magnitude

[12], SEFDM pilots are tested in a static channel to assess the MSE performance. However, in this work, for the same channel, MSE is examined to evaluate the efficacy of SEFDM and OFDM pilots utilization with channel estimation in frequency domain. The static frequency selective channel is

$$
\begin{aligned}
h(t)=0.8765 \delta(t)- & 0.2279 \delta\left(t-T_{s}\right)+0.1315 \delta\left(t-4 T_{s}\right) \\
& -0.4032 \exp (j \pi / 2) \delta\left(t-7 T_{s}\right) . \quad(7)
\end{aligned}
$$

The MSE is evaluated for different compression factors ( $\alpha=$ $1,0.9,0.8,0.7)$ as

$$
M S E=\frac{1}{n} \sum_{i=0}^{n-1}\left(\hat{h}_{i}-h_{i}\right)^{2} .
$$

From Fig. (4), it is clear that the frequency-domain OFDM channel estimation outperforms SEFDM channel estimation, as it results in lower MSE due to the self interference created between subcarriers in SEFDM. Furthermore, as expected, the channel estimation accuracy degrades by decreasing $\alpha$, because with more compression the ICI becomes dominant.

The channel estimation is used to equalize the effect of the channel on the received symbols before detection. The next section looks into the equalization scheme implemented in this work.

\section{B. Channel Equalisation Performance}

The channel equalization is examined for the case of OFDM pilots. As mentioned before, better frequency domain channel estimation can be achieved via OFDM pilot utilization compared to SEFDM, however, a reduction in spectral efficiency (SE) is the drawback, because OFDM pilot has a lower data rate compared to SEFDM pilot with the same frequency spacing. Error vector magnitude (EVM) is measured after channel equalisation using the estimated channel characteristics via OFDM pilot employment. EVM is a figureof-merit used to evaluate the distortion in the received signal introduced by the distortion factors, such as noise and amplitude distortion. In Fig.(5), EVM performances are depicted for different modulation schemes (BPSK, QPSK and 16QAM) with various compression factors $\alpha=1,0.9,0.8,0.7$, where $\alpha=1$ is the case of OFDM. From the EVM curves, it is clear that OFDM outperforms SEFDM as it has lower EVM due to the self-created ICI in SEFDM. The distortion introduced in this system is shown to have, roughly, the same effect on the three modulation schemes.

In addition, Fig. (5) shows the constellation diagram of the equalised received symbols for both OFDM and SEFDM $\alpha=0.8$. It is obvious that the received OFDM symbols are equalised successfully to retrieve the original constellation diagrams contaminated with noise. On the other hand, the equalised SEFDM symbols have a distorted constellation diagram although OFDM pilot symbols are used to estimate the channel characteristics due to the ICI.

The frequency domain channel estimation and equalisation in SEFDM systems reduces the computational complexity compared to the previous implemented time-domain channel estimation/equalisation in [12] with a similar performance. The reason behind this is that the computational complexity of matrix inversion increases exponentially with the number of subcarriers $N$ in time domain scheme, however, in the frequency domain scheme no matrix inversion is required and the complexity increases linearly by $N$.

\section{CONCLUSION}

This work presents the deign and practical implementations of baseband generation, signal assembly, signal decoding and a new frequency-domain channel estimation and equalisation method for SEFDM where subcarriers are compressed to reduce utilized bandwidth and increase SE. Due to the ICI introduced by this compression in SEFDM, a degradation in system performance is noticed when channel estimation via SEFDM pilot to find CSI is used directly.

The experimental work reported shows the feasibility of using SEFDM pilots when ICI is taken into consideration at the receiver side while computing the channel parameters for equalization. However, OFDM pilots employment for estimation outperforms that for SEFDM, but still, this may be considered as a reasonable price for the bandwidth savings in SEFDM. Such experimental results were obtained by testing signals over an LTE channel generated by a VR5-channel emulator operating at a frequency of $2 \mathrm{GHz}$. 
Furthermore, the effect of channel equalisation after estimation is examined by EVM figure-of-merit parameter. Finally, it is shown that this method reduces the complexity of previous work based on channel equalization and estimation in time domain, making this an efficient method for real-time systems implementation.

\section{ACKNOWLEDGMENT}

This work was part funded by two EPSRC grants "Impact Acceleration Discovery to Use" and EP/P006280/1: MARVEL. The work was also supported by National Instruments and a donation of the LTE FPGA core through the Xilinx University Donation program. We are grateful for UCL's studentship for Waseem Ozan and Hedaia Ghannam PhD studies. The work was also supported in part by the EU Funded 5G Exchange Innovation Project, www.5gex.eu.

\section{REFERENCES}

[1] 3GPP TR 36.912 v.13.0.0, Feasibility study for further advancements for E-UTRA (LTE-Advanced), Rel. 13, Dec. 2015.

[2] J. Andrews, S. Buzzi, W. Choi, S. Hanly, A. Lozano, A. Soong, and J. Zhang, What will 5G be? Selected Areas in Communications, IEEE Journal on, vol. 32, no. 6, pp. 10651082, June 2014.

[3] W. Xiang, K. Zheng, and X. Shen, Key Enabling Technologies for 5G Mobile Communications. Springer, 2016.

[4] F.-L. Luo and C. Zhang, Signal Processing for 5G: Algorithms and Implementations. Wiley, 2016.

[5] H. A. U. Mustafa, M. A. Imran, M. Z. Shakir, A. Imran, and R. Tafazolli, Separation framework: An enabler for cooperative and D2D communication for future 5G networks, IEEE Communications Surveys Tutorials, vol. 18, no. 1, pp. 419445, Firstquarter 2016.

[6] M. Rodrigues and I. Darwazeh, A spectrally efficient frequency division multiplexing based communications system, in Proc. 8th Int. OFDM Workshop, Hamburg, 2003, pp. 4849.

[7] I. Kanaras, A. Chorti, M. R. D. Rodrigues and I. Darwazeh, "Spectrally Efficient FDM Signals: Bandwidth Gain at the Expense of Receiver Complexity," 2009 IEEE International Conference on Communications, Dresden, 2009, pp. 1-6.

[8] J. Mazo, Faster-than-Nyquist signaling, Bell Syst. Tech. J, vol. 54, no. 8, pp. $14511462,1975$.

[9] J. B. Anderson, F. Rusek and V. wall, "Faster-Than-Nyquist Signaling," in Proceedings of the IEEE, vol. 101, no. 8, pp. 1817-1830, Aug. 2013.

[10] W. Ozan, K. Jamieson and I. Darwazeh, "Truncating and oversampling OFDM signals in white Gaussian noise channels," 2016 10th International Symposium on Communication Systems, Networks and Digital Signal Processing (CSNDSP), Prague, 2016, pp. 1-6.

[11] S. Isam and I. Darwazeh, "Robust channel estimation for Spectrally Efficient FDM system," 2012 19th International Conference on Telecommunications (ICT), Jounieh, 2012, pp. 1-6.

[12] T. Xu and I. Darwazeh, "Transmission Experiment of Bandwidth Compressed Carrier Aggregation in a Realistic Fading Channel," in IEEE Transactions on Vehicular Technology, vol. 66, no. 5, pp. 4087 4097, May 2017.

[13] H. Ghannam and I. Darwazeh, Robust channel estimation methods for spectrally efficient FDM systems, in VTC 2018 workshop; Technology Trials and Proof-of-Concept Activities for $5 G$ and Beyond 2018 (TPoC5G 2018) (Accepted), June 2018.

[14] S. Isam and I. Darwazeh, "Characterizing the intercarrier interference of non-orthogonal Spectrally Efficient FDM system," 2012 8th International Symposium on Communication Systems, Networks and Digital Signal Processing (CSNDSP), Poznan, 2012, pp. 1-5.

[15] I. Kanaras, A. Chorti, M. R. D. Rodrigues and I. Darwazeh, "A Fast Constrained Sphere Decoder for Ill Conditioned Communication Systems," in IEEE Communications Letters, vol. 14, no. 11, pp. 9991001, November 2010

[16] T. Xu and I. Darwazeh, A soft detector for spectrally efficient systems with non-orthogonal overlapped sub-carriers, textitCommunications Letters, IEEE, vol. 18, no. 10, pp. 18471850, Oct 2014.

[17] J. Huang, Q. Sui, Z. Li and F. Ji, "Experimental Demonstration of 16QAM DD-SEFDM With Cascaded BPSK Iterative Detection," in IEEE Photonics Journal, vol. 8, no. 3, pp. 1-9, June 2016.

[18] K. Park, H. Kim, A. Lee, D. Kang and W. Oh, 'Iterative frequencydomain inter-carrier interference cancellation for coded SEFDM," in Electronics Letters, vol. 53, no. 19, pp. 1333-1335, 9142017.
[19] A. Rashich, A. Kislitsyn, D. Fadeev and T. Ngoc Nguyen, "FFT-Based Trellis Receiver for SEFDM Signals," 2016 IEEE Global Communications Conference (GLOBECOM), Washington, DC, 2016, pp. 1-6.

[20] H. Ghannam and I. Darwazeh, "SEFDM over satellite systems with advanced interference cancellation," in IET Communications, vol. 12, no. 1 , pp. 59-66, 152018

[21] Evolved Universal Terrestrial Radio Access (E-UTRA); Base station radio transmission and reception, 3GPP TS 36.104 V10.2.0, May 2011.

[22] T. Schmidl and D. Cox, Robust frequency and timing synchronization for OFDM, Communications, IEEE Transactions on, vol. 45, no. 12, pp. 16131621, Dec 1997. 